\title{
DETERMINATION OF THE HEIGHT OF HARD X-RAY SOURCES IN THE SOLAR ATMOSPHERE BY MEASURE- MENT OF PHOTOSPHERIC ALBEDO PHOTONS
}

\author{
JOHN C. BROWN* and H. F. VAN BEEK \\ Space Research Laboratory of the Astronomical Institute, Utrecht, The Netherlands
}

\begin{abstract}
Summary**. The importance and difficulties of determining the height of hard $\mathrm{X}$-ray sources in the solar atmosphere, in order to distinguish source models, have been discussed by Brown and McClymont (1974) and also in this Symposium (Brown, 1975; Datlowe, 1975). Theoretical predictions of this height, $h$, range between $\lesssim 10^{3}$ and $10^{5} \mathrm{~km}$ above the photosphere for different models (Brown and McClymont, 1974; McClymont and Brown, 1974). Equally diverse values have been inferred from observations of synchronous chromospheric EUV bursts (Kane and Donnelly, 1971) on the one hand and from apparently behind-the-limb events (e.g. Datlowe, 1975) on the other.
\end{abstract}

Direct resolution of the height of a source at the limb for sources at the low end of this height range (corresponding to angles $\zeta \lesssim 1$ ". 4 at the Sun) is certainly impossible for any planned hard X-ray heliograph while, even for sources in the $10^{4}-2 \times 10^{4} \mathrm{~km}$ range $(\zeta \simeq 14$ ".-28"), this method does not distinguish low sources exactly at the limb from high sources near the limb. Thus it is of interest to consider whether heights might be inferred for sources on the disk and particularly for those at low altitudes. This is made potentially possible only by virtue of the fact that the dense photosphere effectively provides a 'mirror' behind the source due to Compton backscattering of X-rays (Tomblin, 1972; Santangelo et al., 1973). Though several means may be considered for utilising this (e.g. Brown et al., 1974) the most promising one in terms of planned instrumentation is, in our view, spatial resolution of the patch of albedo $\mathrm{X}$-rays behind the primary source. For simplicity here we consider only the case of a small primary source (diameter $\lesssim 10^{\prime \prime}$ ) at the disc center, for which the albedo patch consists of circular isobrightness contours out to the source's solar horizon at a distance $r \simeq \sqrt{ } 2 R h$ ( $R=$ solar radius) corresponding roughly to angular radii of $50^{\prime \prime}, 160^{\prime \prime}$ and $350^{\prime \prime}$ for $h=10^{3}, 10^{4}$ and $5 \times 10^{4} \mathrm{~km}$ respectively (i.e. $\zeta \sim 1 " 3,13^{\prime \prime}$ and $65^{\prime \prime}$ ). Clearly, therefore, observation of this patch requires much less resolution than needed for the source height itself - the essential advantage of the method. Furthermore, the evidence is that the primary source does indeed have a small horizontal extent (Takakura et al., 1971), so the method is unlikely to be vitiated by obscuration of the albedo distribution by the primary emission.

Actual detection of the albedo patch depends on the capabilities of the hard X-ray heliograph employed. In particular, the brightness of the patch is easily shown to drop

* On leave from: Dept. of Astronomy, University of Glasgow, Scotland.

** Paper to appear in Astronomy and Astrophysics. 
off from the subsource point roughly like

$$
d I\left(\text { cts per } \mathrm{cm}^{2} \text { per square arc sec }\right)=\frac{f I_{0} \zeta}{2 \pi\left(\varrho^{2}+\zeta^{2}\right)^{3 / 2}},
$$

where $\varrho$ is the angular distance from the subsource point, $I_{0}$ is the primary source photon flux (here assumed isotropic) and $f$ is the total backscattered fraction. Distribution (I) has to be compared with the instrumental response profile to a point source to determine the actual angular radius $\varrho_{0}$ over which the albedo is detectable. The Hard X-ray collimating heliograph under development in Utrecht forms real-time pictures in the form of a $4.3 \times 4.3$ array of square elements of $8^{\prime \prime} \times 8^{\prime \prime}$ each in 5 energy hands from $3.5-20 \mathrm{keV}$. Each element has a sensitive area of $4 \mathrm{~mm}^{2}$. The highest (15-20 keV) channel of this instrument is about ideal for observing the albedo, being above the range where $f$ is reduced by photospheric absorption (Santangelo et al., 1973 ) but low enough to maintain reasonable count rates. Figure 1 shows the angular

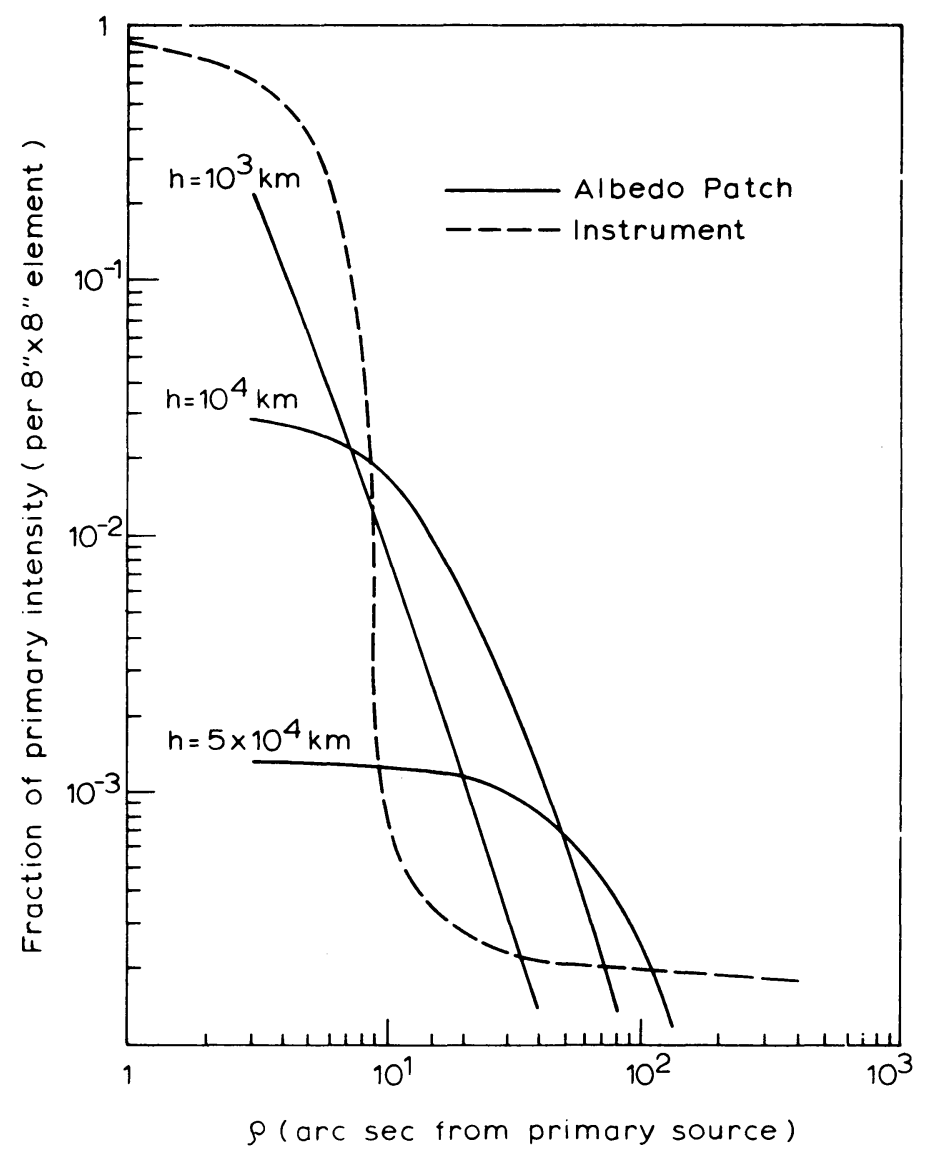

Fig. 1. Albedo brightness distribution as a function of the angular radius $\varrho^{\prime \prime}$ from a primary source at the disc center, for various source heights $h$, compared to the instrumental profile for a point source. 
response profile of this instrument to a point source, mainly due to collimator characteristics, compared to the albedo profile for $h=10^{3}, 10^{4}$, and $5 \times 10^{4} \mathrm{~km}$. It is evident that the albedo patch can indeed be measured with this instrument for such source heights (see also Table I).

Finally it is necessary to consider the integration time necessary to determine the albedo distribution above the statistical noise which depends of course, on the actual primary source intensity. However, even for a fairly modest event, with $I_{0}=10^{3}$ photons $\mathrm{cm}^{2} \mathrm{~s}^{-1}$ in the $15-20 \mathrm{keV}$ range, it is found that a $30 \mathrm{sec}$ integration time will give ample precision in the albedo measurement for $h \leqslant 5 \times 10^{4} \mathrm{~km}$ (Table I).

\section{TABLE I}

Visibility of X-ray albedo above the instrument profile

Source Height

$h(\mathrm{~km}) \quad \xi$

$10^{3}$

$10^{4}$

$5 \times 10^{4}$
Albedo visible above instrument profile over

Radius $Q_{0}$

No. of image elements
Estimated variance in albedo counts after $30 \mathrm{~s}$ integration for a flare producing $10^{3}$ photons $\mathrm{cm}^{-2} \mathrm{~s}^{-1}$ between 15 and $20 \mathrm{keV}$

$16 \%$

$6 \%$

$10 \%$

\section{References}

Brown, J. C.: 1975, This volume, p. 245.

Brown, J. C., McClymont, A. N., and McLean, I. S.: 1974, Nature 247, 448.

Brown, J. C. and McClymont, A. N.: 1974, Solar Physics, in press.

Datlowe, D. W.: 1975, This volume, p. 191.

Kane, S. R. and Donnelly, R. F.: 1971, Astrophys. J. 164, 151.

McClymont, A. N. and Brown, J. C.: 1974, in preparation.

Santangelo, N., Horstman, H., and Horstman-Moretti, E.: 1973, Solar Phys. 29, 143.

Tomblin, F. F.: 1972, Astrophys. J. 121, 377.

Takakura, T., Ohki, K., Shibuya, N., Fujii, M., Matsuoka, M., Miyamoto, S., Nishimura, J., Oda, M., Ogawara, Y., and Ota, S.: 1971, Solar Phys. 16, 454. 